\title{
Person Identification Using Gait
}

\author{
A. Hayder, J. Dargham, A. Chekima, and G. M. Ervin
}

\begin{abstract}
Principal Component Analysis (PCA) with and without Radon Transform (RT) are applied for gait recognition purposes. The Radon Transform is used to detect features within an image and PCA is used to the reduce dimension of the images without much loss of information. The side view of slow walk, fast walk and carrying a ball walk have been selected from the CMU MoBo database for experimental purposes. The two techniques experimental result achieved equal recognition rates (EER) of $\mathbf{8 5 . 4 0 \% , 7 8 . 0 7 \%}$ and $\mathbf{9 0 . 0 5 \%}$ for RT with PCA and $85.18 \%, 80 \%$, and $89.90 \%$ for PCA only for slow walk, fast walk and carrying a ball walk respectively.
\end{abstract}

Index Terms--Gait Recognition, PCA, Radon Transform, Segmentation, Silhouette Extraction.

\section{INTRODUCTION}

Gait recognition system can be classified depending on the sensors used into three groups namely; motion vision based, wearable sensor based and floor sensor based. The motion vision can be divided into two groups namely; appearance based methods and model based methods. The appearance based method can be also subdivided in two types; state space methods and spatio-temporal methods. The classification of the recognition system is shown in "Fig 1", [1-7].

Biometric gait recognition refers to verifying or identifying persons using their walking style. Human recognition based on gait is relatively recent compared to other biometric approaches such as fingerprint, iris, facial etc.

The wearable sensors and floor sensors systems are also able to identify persons but in different conditions compared to motion vision technique. The wearable sensors technique needs to carry necessary sensors which enable to measure the different walk styles. The sensors can be set on any part of the body according to the sensors characteristics to get gait data to match up with training dataset. The sensors may be set up on hip, legs, arms or other parts of the body [8].

The floor sensors are put into the floor or on the floor which enable to detect the required measurement. The most important point is to match up testing dataset with training dataset to identify the subjects. Both systems are useful for access control such as office, airport, mega mall and other restricted places [9].

"Fig 2" shows a typical motion vision based system flow chart, while "Fig 3" shows a general block diagram of gait recognition system.

Motion vision can be used for surveillance, access control, detection and other monitoring purposes. The most important advantage is that person walking image can be captured from long distance and the image is then processed

Manuscript received November 4, 2010; revised July 6, 2011 with low resolutions [7].

In this paper, we focus on two different techniques Principle Component Analysis (PCA) only and PCA with radon transform (RT) on machine vision for gait recognition purposes.

In the next section, the literature review will be discussed briefly. The system design is described in section III, while the results are discussed in section IV. Conclusion and future directions are given in section $\mathrm{V}$.

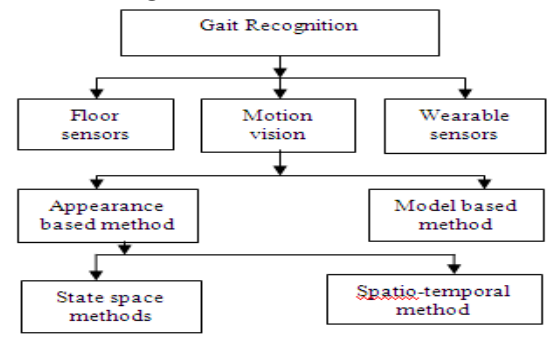

Fig 1: Classification of gait recognition system $[1,2]$

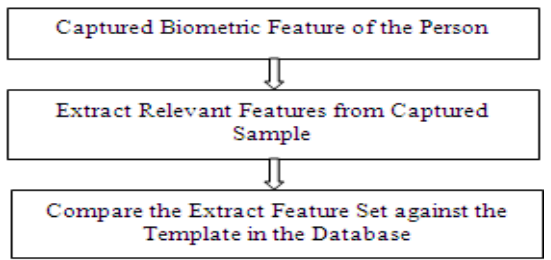

Fig 2: Motion vision based system flow chart [1, 3]

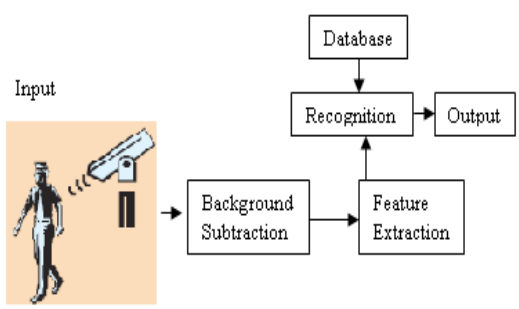

Fig 3: General block diagram of a gait recognition/authentication system [5]

\section{LITERATURE REVIEW}

Huang and Boulgouris [10] proposed a gait recognition system that uses multiple views. They have used six views according to Motion of Body (MoBo) database. They combined the gait cycles which resulted in an improved recognition rate of $96 \%$.

Guo and Tian [11] proposed a gait recognition method based on anatomical knowledge. They applied Hidden Markov Model for the experiment purposes. At first, they extracted the silhouette applying morphological operation and selected gait period. For the experiment, they used CMU gait database and selected three walking styles gait cycles which are fast, slow and carrying a ball for identification purposes.

Sharmila and kirubakaran [12] developed a method to extract human gait feature automatically even in low resolution. They applied two techniques for silhouette 
extraction called Image Based Gait Recognition and Formula Based Gait Recognition in free motion features from video sequence. The Image based gait technique can deal with clothing, lighting, segmentation and tracking. The formula based approach deals with height and stride parameters of walking gait. They used their own video frame and applied it for Identification purposes.

Honggui and Xingguo [13] proposed dimension reduction technique for gait images. They found that the Gaussian technique is better than the normal background subtraction technique. They also applied gait alignment technique. The different styles of gait cycles have been applied for test and finally Fast Fourier Transform (FFT) module apply to 1D Locally Linear Embed (LLE) for gait recognition purposes. They applied CMU MoBo gait database and achieved 92\% recognition rate in rank 5.

Qiong et al. [14] proposed gait recognition based on PCA and Linear Discriminate Analysis LDA. PCA is mainly used for dimensional reduction technique and LDA is performed to optimize the pattern class. For the experiment, they used their own database and they achieved better recognition rate from PCA compared to (LDA). In this paper, we discus and compare the result about PCA with RT and PCA without RT technique for gait recognition purposes.

\section{SYSTEMS DESIGN}

\section{A. Silhouette Extraction}

In gait recognition, silhouette is defined as a region of pixels of the walking person. Silhouette extraction mainly focuses on segmenting the human body. The silhouette extraction process is shown in "Fig 4".

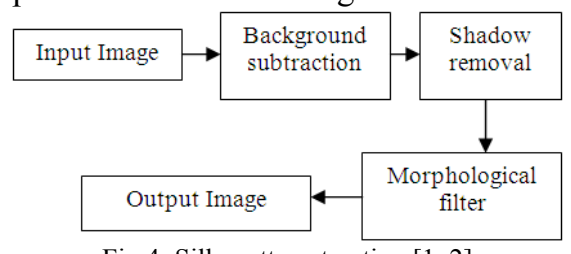

Fig 4: Silhouette extraction $[1,2]$

Each of the frames in the image sequence is subtracted from a background model of the respective image sequence. If the pixel value of each frame is not the same with the pixel value of the background, the pixel is marked as region of silhouette [15]. A sample of silhouette extraction process is shown in "Fig 5".

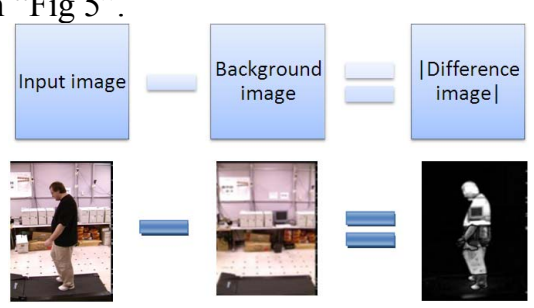

Fig 5: Sample of extraction silhouette process

To remove shadow from the difference image, a threshold value is applied to the difference images. The difference image map is first analyzed by generating the intensity histogram of the image so that the pixels distribution along the image can be represented clearly and in an effective way according to an applied threshold value. The threshold must be suitable so that the foreground image is neither undersegmented nor over-segmented. To produce better silhouette from the difference image, two values of threshold T1 and $\mathrm{T} 2$ are used. This will produce two different images which are under-segmented and over-segmented image. T1 is used to produce the under-segmented image while $\mathrm{T} 2$ is used to produce the over-segmented image. Under-segmentation and over-segmentation purpose is to produce first and second reliable silhouette respectively.

To remove noises produced during segmentation of silhouette, morphological filters are used. The main components of morphological filters that are used in the system are morphological opening, morphological closing and area thresholding through connected component labelling. Morphological opening and closing consist of two basic morphological operations which are erosion and dilation. Morphological opening performs erosion first, followed by dilation, while morphological closing is vice versa [1 2 16]. "Fig 6" shows samples of morphological filters images.

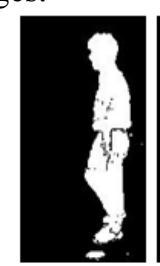

(a)

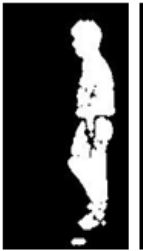

(b)

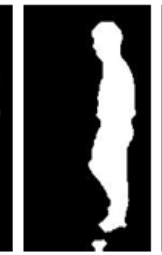

(c)

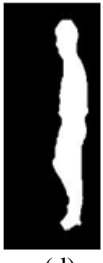

(d)
Fig 6: Example of morphological filters in action (a) Input silhouette from shadow removal,

(b) Morphological opening, (c) Morphological closing, (d) Area thresholding.

\section{B. PCA with RT and PCA only}

The Radon Transform is one of the powerful techniques used to identify features within an image. Let $\mathrm{L}(\mathrm{m}, \mathrm{n})$ be an image function, the Radon Transform is defined as:

$$
F(\rho, \theta)=\int_{-\infty}^{\infty} f(\rho \cos \theta-x \sin \theta, \rho \sin \theta+x \cos \theta) d x
$$

where, $\mathrm{x}$ is defined as an integral along a line through the image, $\theta$ is the angle and $\rho$ is the distance of the line from the origin of the coordinate system as shown in "Fig 7".

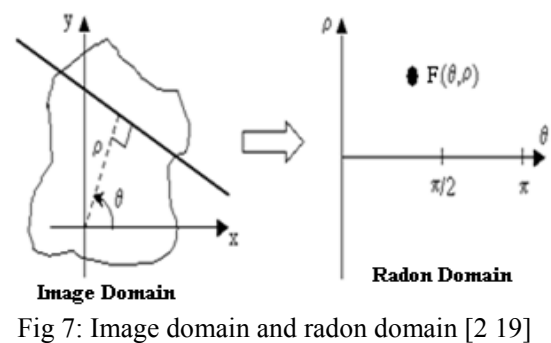

"Fig 8" shows the block diagram of PCA with Radon Transform. Radon transform will be applied on the image to compute its $2 \mathrm{D}$ projection image along angles varying from 00 to 1800 . The result of the projection is the sum of the intensities of the pixels in each direction. All the projections of the image are concatenated to form 1D Radon Transform vector. The 1D Radon Transform vector for all training images are computed. PCA is then applied on the collection of 1D Radon Transform vector to produce a low dimensional feature vector.

Principal Component Analysis (PCA) is a statistical analysis of data for finding the principal components or eigenvectors and eigenvalues on which the data is most likely to vary. PCA is used in identifying patterns in data, 
and expressing the data in such a way as to highlight their similarities and differences. Since patterns in data can be hard to find in data of high dimension, where the data cannot be graphically represented, PCA is a powerful tool for analyzing data.

The main advantage of PCA is that once these patterns in the data have been found, these patterns are compressed by reducing the number of dimensions, without much loss of information. This technique was mainly used in image compression, but some researchers had applied PCA in their pattern recognition algorithm and proven to be successful [1 18]. Dimensionality reduction is vital to the recognition purposes because the size of recognition matrices can be large and very computationally expensive or infeasible.

PCA is used to simplify the data structure and still account for as much of the total variation in the original data as possible [l 18 18]. Several theoretical details parameters which are mean image, zero mean matrix, covariance matrix, eignvectors and eigenvalues, and sorting of eigenvalues are used [17]. The Empirical mean is defined as in equation (2)

$$
u[m]=\frac{1}{N} \sum_{n=1}^{N} X[m, n]
$$

where, the empirical mean is taken along each dimension $\mathrm{m}=1,2, \ldots, \mathrm{M}$. Here the mean vector $\mathbf{u}$ has a dimension of M x 1. To calculate the deviations from the mean, subtract the empirical mean vector $\mathbf{u}$ from each column of the data matrix $\mathrm{X}$ which is defined as $B=X-u h$, where mean subtracted data $\mathrm{B}$ is an $\mathrm{M} \times \mathrm{N}$ matrix $\mathrm{B}$ and $\mathrm{h}$ is a $1 \mathrm{xN}$ row vector of all 1 's.

The covariance matrix, $\mathrm{C}$ is expressed by equation (3)

$$
C=E[B \otimes B]=E\left[B \cdot B^{*}\right]=\frac{1}{N} \sum B \cdot B^{*}
$$

where, $\mathrm{B}$ is the expected value, and $\otimes$ is the outer product. The computed matrix $\mathrm{V}$ of eigenvectors which is diagonalizable covariance matrix $\mathrm{C}$ is defined as: $\mathrm{V}^{-1} \mathrm{CV}=$ $\mathrm{D}$, where $\mathrm{D}$ is the diagonal matrix of eigenvalues of $\mathrm{C}[14$, $15]$.

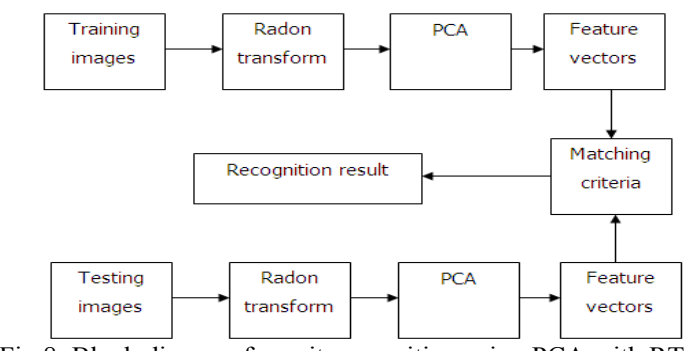

Fig 8: Block diagram for gait recognition using PCA with RT [2]

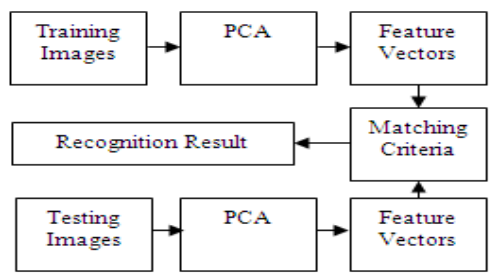

Fig 9: Block diagram for gait recognition using PCA only [1]

"Fig 9" shows the block diagram for gait recognition using PCA method used for this paper. 2-D gait images are concatenated to form 1-D image vectors. Zero mean 1-D training images set are computed. PCA is then applied on the collection of 1-D zero-mean images set vector to produce a low-dimensional features vector.

\section{Training and Testing Database}

The CMU MoBo gait database has 25 subjects with four kinds of walking styles and six views for each waking style [18]. A total of 13 subjects for training have 6729 frames rearranged silhouettes. Each subject has different number of frames for each cycle for all three walking styles; slow walk, fast walk, and carrying a ball walks. The total numbers of testing frames are 2714 frames. The testing frames will be used to test the recall capability of the gait recognition system. Each subject has a minimum of 14 cycles and rearranged as 10 cycles for training and 4 cycles for testing and each gait cycle has approximately 20 frames. In this paper, the total number of images used for three walking styles are 6729 for training, 2714 for testing for PCA with RT and PCA only techniques. Another 12 subjects called unknown (630 frames) which have also one cycle for all three walking styles to prepare for reject capability of the system for both techniques. "Fig 10" shows the design of testing and training dataset. All the images have been resized from $486 * 640$ to $152 * 200$ size.

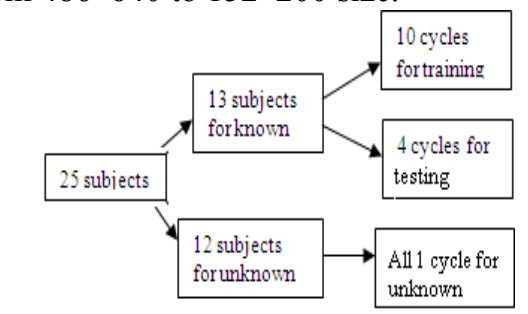

Fig 10: Design of training and testing dataset $[1,2]$

\section{Matching Criteria}

The Euclidean distance is used for measuring purposes. If the Euclidean distance between frame A and frame B in the training database is smaller than a fixed threshold value $\mathrm{T}$, then frames A and B are considered to be the same subject. Threshold $\mathrm{T}$ is the largest Euclidean distance between any two images of the subject in the training database, divided by a threshold tuning value (Tcpara) as given in equation (5). The threshold is defined as

$$
T=\frac{[\max \langle\|\Omega j-\Omega k\|\rangle]}{\text { Tcpara }}
$$

where, Tcpara is a tuning value; $\mathrm{j}, \mathrm{k}=1,2,3 \ldots, \mathrm{N}$. N is the total number of training images and $\Omega$ are the reduced dimension images for a given subject of the frames. A sample of the Euclidean distance to verify frame to frame matching for one subject as shown in "Fig 11". It can be seen from "Fig 11" that each frame displays different magnitudes on one complete cycle. It is easy to select a gait cycle by applying Euclidean distance method. The gait cycle can also be obtained manually.

In the algorithm, two performances matrices have been measured which are namely recall and reject. For recall, if a test image is correctly identified to an image of the same person from the training database, it is called Correct Classification. However, if the test image is incorrectly matched with another subject images it is called False Acceptance. If an image from the training database is rejected by the system then, it is called False Rejection. For reject, if any test frame from the unknown set cannot be identified by system then it is called Correct Classification. If the test image can be detected by system then it is called False Acceptance. 


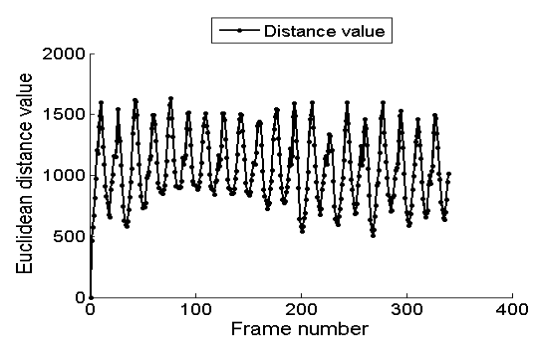

Fig 11: Sample of gait cycle using Euclidean distance

\section{E. Threshold Tuning Parameter}

The value of the threshold tuning parameter can be used to tune the performance of the system to have either high correct recall with high false acceptance rate for application such as boarder monitoring or high correct rejection rate for unknown persons for application such as access control. For this work, the threshold tuning parameter was set so that the system has equal correct recall rate and correct rejection rate The Tcpara (threshold) value mentioned that was chosen for each system is shown "Fig 12" to "Fig 17" and it is observed the each walking style has different Tcpara value.

\section{Result AND Discussion}

"Fig 18" shows experimental performance using PCA with RT and PCA only for three walking styles. The case where the recall correct classification is equal to reject correct classification is called EER. For this work, the EER achieved $85.40 \%, 78.07 \%$ and $90.05 \%$ for PCA with RT method and $85.18 \%, 80 \%$, and $89.80 \%$ for PCA only method for the three walking styles slow walk, fast walk and carrying a ball walk respectively. If we set Tcpara value to 1 that the best recall correct classification rate attained above $95 \%$ for the two methods for all three walking styles while the reject correct classification rate is $0 \%$. It can be seen from "Fig 12" to "Fig 17" then the recall correct classification rate is decreasing as Tcpara value increases, while the reject correct classification rate is increasing as Tcpara value increases. However, for each walking style, FAR and FRR might be calculated from both systems according to the recognition rate. The FAR for both recall and rejection are decreases as Tcpara value increases. So, different walking styles have different threshold value for the system.

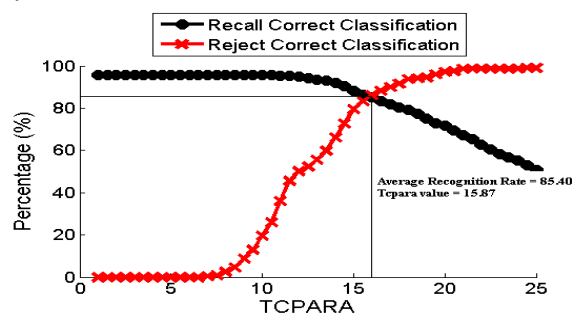

Fig 12: Recognition result for slow walk using PCA with RT

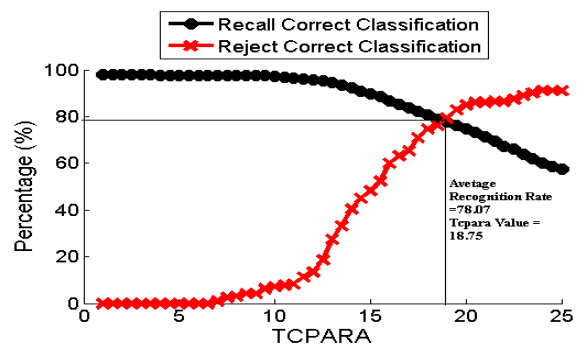

Fig 13: Recognition result for fast walk using PCA with RT

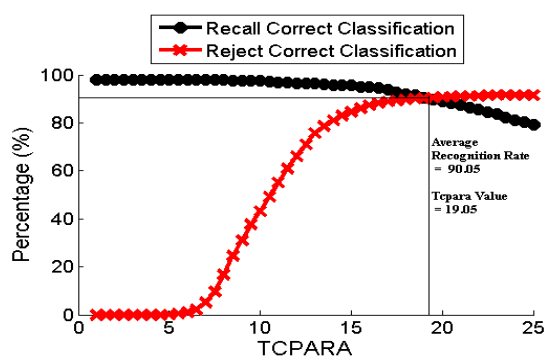

Fig 14: Recognition result for carrying a ball walk using PCA with RT

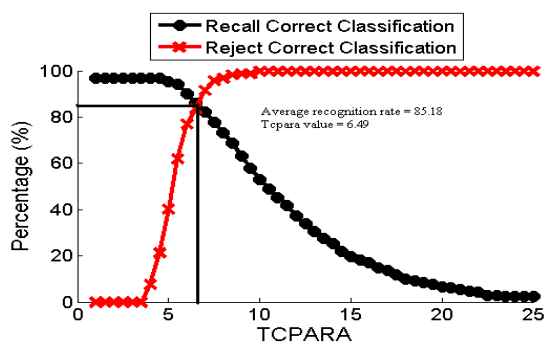

Fig 15: Recognition result using PCA for slow walk

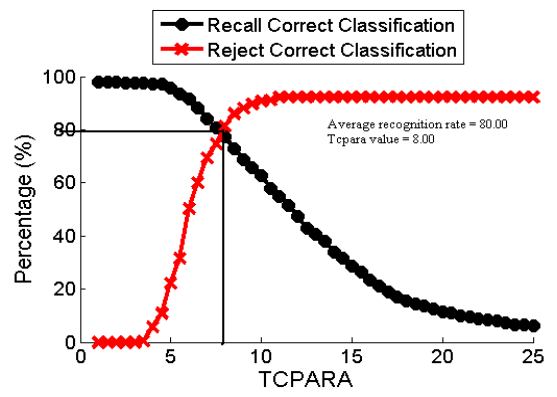

Fig 16: Recognition result using PCA for fast walk

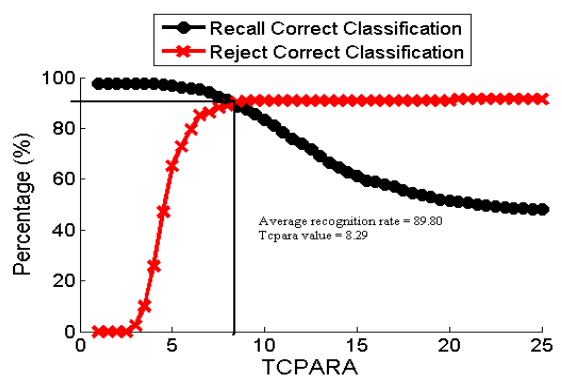

Fig 17: Recognition result using PCA for carrying a ball walk

Equal recognition rate for three Walk Styles using PCA with and without Radon Transform

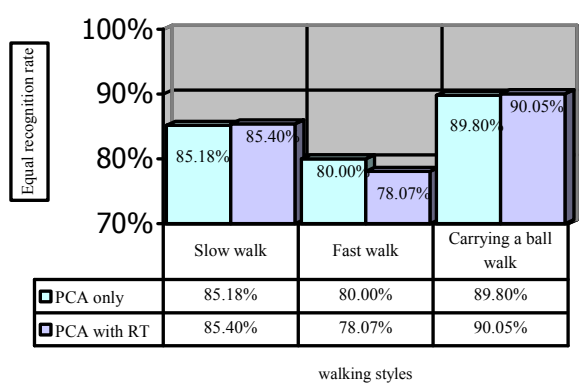

Fig 18: Equal Error Rate for three Walk Styles using PCA with and without Radon Transform

\section{A. Comparison between PCA with RT and PCA only}

"Fig 18" shows that PCA with RT gives slightly better result compared to PCA only technique except for fast walking style. If we compare the recognition rate among three the walking styles, the carrying a ball walk style presents better result for both methods. The fast walk style presents low recognition rate compared to slow walking and carrying a ball walking style for both techniques, but the 
slow walk recognition rate is significantly better than the PCA while RT. For the fast walking style, PCA technique obtained slightly better result than using PCA with RT method. We observed that slow walk, fast walk, and carrying a ball walk EER achieved approximately identical by applying both techniques. If we set TCPARA value to 1 for all three walking styles then it will achieve a high recognition rate of $95 \%$ for both techniques.

B. Comparison of our results to other researchers' results

We have compared our system with other methods reported in published papers as shown Table 1. Lei et al. [22] presented gender recognition system and acquired recognition rate $95.67 \%$ by applying RT and Recursive Component Analysis (RCA) methods. Qiong et al. [14] applied PCA and LDA methods and attained above 95\% recognition rate. Murat [23] employed PCA method for experiment and achieved $100 \%$ recognition rate. For the experiment purpose, he used CMU MoBo gait database. Siva and Mathivanan [24] applied LDA and RT methods for the experiment and achieved above $95 \%$ recognition rate. In the above mentioned papers the researchers have used different existing gait database. The referred papers have rearranged gait database in different conditions compared to our rearranged gait database described in section III (c). "Fig 19" shows output of same correct matching frames. "Fig 19" displayed that matching frame "Test[47]Train[114]" which correct matching training dataset. However, the matching frames can be identified manually from output matching frames.

TABle 1: Comparison Of SeVeral Methods Based Gait Recognition ON DifFERENT GaIT DATABASE

\begin{tabular}{|l|l|}
\hline \multicolumn{1}{|c|}{ Methods } & Recognition Rate \\
\hline RT and RCA [22] & $95.67 \%$ \\
\hline PCA and LDA [14] & above $95 \%$ \\
\hline PCA [23] & $100 \%$ \\
\hline LDA and RT [24] & Above $95 \%$ \\
\hline Our PCA method & above $\mathbf{9 5 \%}$ \\
\hline Our PCA and RT method & above 95\% \\
\hline
\end{tabular}

\section{CONCLUSION}

In this paper, a gait recognition style based on PCA with RT and only PCA techniques are proposed. The proposed systems were presented adequate results while applying CMU MoBo gait database applied for the experiments. The best recognition above $95 \%$ is achieved for PCA with RT and PCA only for all three walking styles. The results were compared with other published papers and reported that the proposed system gives efficient result. Some of researchers presented better recognition rate of walking style in different gait database conditions.

\section{ACKNOWLEDGEMENT}

This work was supported by University Malaysia Sabah (UMS) postgraduate fund (GPS0002-TK-1/2009). The authors also would like to thank the CMU MoBo gait database authors for providing the gait database for research purposes.
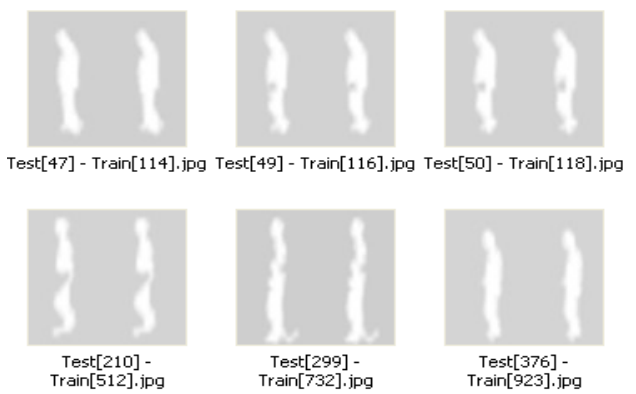

(a) Slow walk using PCA
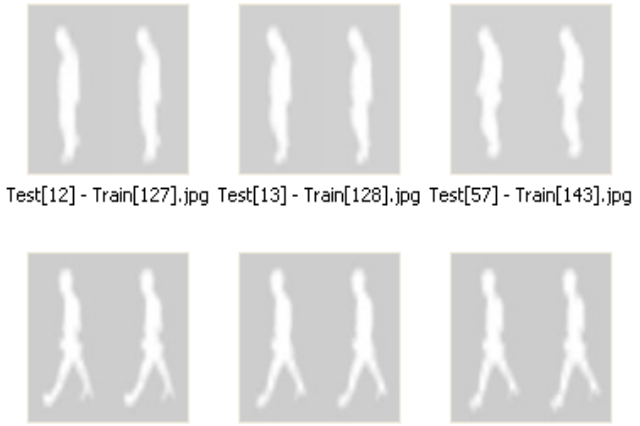

Test[63] - Train[273].jpg Test[64] - Train[274].jpg Test[65] - Train[275].jpg

(b) Fast walk using PCA

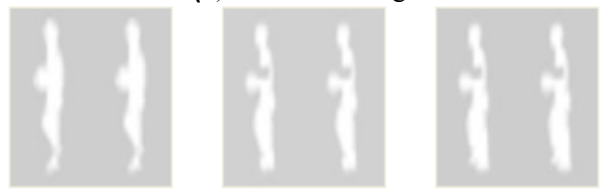

Test[75] - Train[185].jpg Test[76] - Train[323].jpg Test[77] - Train[324].jpg

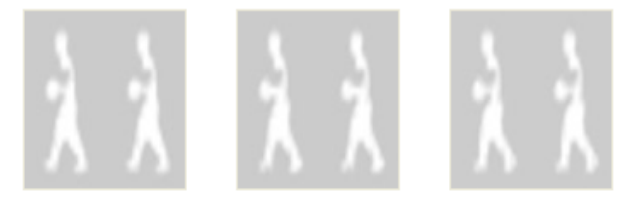

Test[83] - Train[330].jpg Test[84] - Train[331].jpg Test[85] - Train[332].jpg (c) Carrying a ball walk using PCA
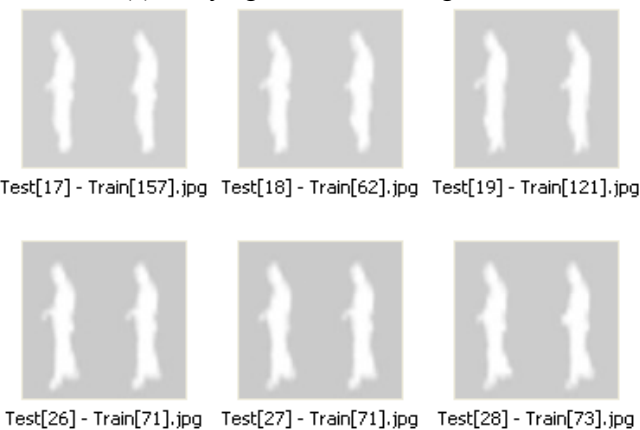

(d) Slow walk using PCA with RT
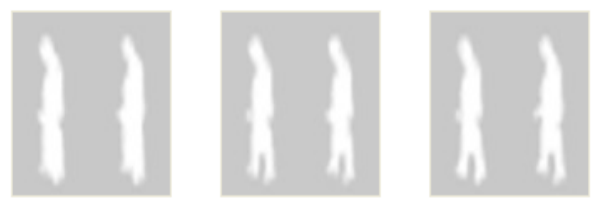

Test[17] - Train[17].jpg Test[18] - Train[132].jpg Test[19] - Train[18].jpg

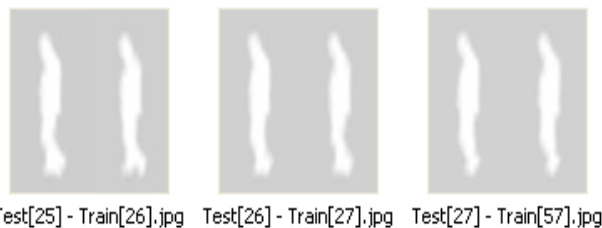

(e) Fast walk using PCA with RT 

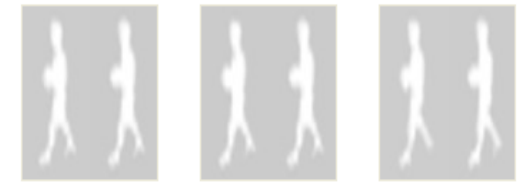

Test[28] - Train[176].jpg Test[29] - Train[176].jpg Test[30] - Train[178].jpg
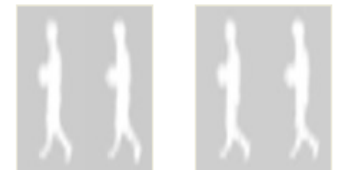

Test[50] - Train[123].jpg Test[51] - Train[125].jpg Test[52] - Train[126].jpg

(f) Carrying a ball walk using PCA with RT

Fig 19: Sample of output of correct matching frames using PCA with and without RT

\section{REFERENCES}

[1] H. Ali, Jamal Dargham, Chekima Ali, Ervin Gobin Moung; "Gait Recognition using Principal Component Analysis", $3^{\text {rd }}$ International Conference on Machine Vision (ICMV), China, Hong Kong, December 28-30, 2010, pp: 539-543.

[2] H. Ali, Jamal Dargham, Chekima Ali, Ervin Gobin Moung; "Gait Recognition using Radon Transform with Principal Component Analysis", $3^{\text {rd }}$ International Conference on Machine Vision (ICMV), China, Hong Kong, December 28-30, 2010, pp:585-589.

[3] L. Feng Liu, W. Jia, and Y. Hai Zhu; "Survey of Gait Recognition". Springer-Verlag Berlin Heidelberg, ICIC 2009, LNAI 5755, 2009, pp: 652-659.

[4] T. B. Moeslund, A. Hilton, V. Kruger; "A Survey of Advances in Vision-Based Human Motion Capture Analysis", Computer Vision and Image Understanding 104, 2006, pp: 90-126.

[5] D. C. Post; 2006; "Gait Analysis Review". www.nd.edu/ dpost/IntSyst/report1.pdf

[6] D. Gafurov; "A Survey Of Biometric Gait Recognition: Approaches, Security and Challenges", NIK-2007 conferences; URL http://www.nik.no/

[7] N.V. Boulgouris, D. Hatzinakos, K.N Plataniotis; "Gait Recognition: A Chlelnging Signal Processing Technology for Biometric Identification", signal processing magazine, IEEE, 2005, pp: 78-90.

[8] L. Rong, Zhou Jianzhong, Liu Ming, Hou Xiangfeng; "A Wearable Acceleration Sensor System for Gait Recognition". Industrial Electronics and Applications, ICIEA, IEEE Conference, 2007, pp 2654-2659.

[9] Lee Middleton, Alex A. Buss, Alex Bazin,Mark S. Nixon; "A Floor Sensor System for Gait Recognition". Proceedings of the IEEE Workshop on Automatic Identification Advanced Technologies, 2005 pp: 171-176.

[10] X. Huang and N.V. Boulgouris, "Gait Recognition using Multiple Views". Proceeding of the IEEE International Conference on Acoustics, Speech, and Signal Processing, 2008, pp: 1705-1708.

[11] Y. Guo, G. Tian, "Gait Recognition Based on Anatomical Knowledge" Intelligent Control and Automation, WCICA 7th World Congress, 2008, pp: $6803-6806$.

[12] D. Sharmila and E. Kirubakaran; "Image and Formula Based Gait Recognition Methods". International Journal of Computer and Electrical Engineering, Vol. 2, No. 2, April, 2010, pp: 381-388.

[13] G. Li, X. Li; 2004, SINOBIOMETRICS: Chinese conference on biometric recognition No5, Chine, vol. 3338, 2010, pp. 671-679.

[14] Q. Cheng, B. Fu, and H. Chen; "Gait Recognition Based on PCA and LDA". Proceedings of the Second Symposium International Computer Science and Computational Technology, Huanshan, P. R. China, 2006, pp: 124-127.

[15] X. Chen; Z. He; D. Anderson; J. Keller; M. Skubic, "Adaptive Silhouette Extraction and Human Tracking In Complex And Dynamic
Environments", Image Processing, IEEE International Conference, 2006, pp: $561-564$

[16] J.L Landabaso., M. Pardàs and L.Q., Xu; "Shadow Removal with Morphological Reconstruction", In Proceedings of Jornades de Recerca en Automàtica, Visió i Robòtica (AVR), Barcelona (Spain), 2004.

[17] P. Toft, 1996, "The Radon Transform Theory and Implementation", Ph.D Thesis, Technical University of Denmark.

[18] M. Miciak; "Radon Transformation And Principal Component Analysis Method Applied In Postal Address Recognition Task", International Journal of Computer Science and Applications, Technomathematics Research Foundation, Vol. 7, No. 3, pp. 33 - 44, 2010 .

[19] "Principle Component Analysis". http://en.wikipedia.org/wiki/Principal_component_analysis

[20] Ralph and S. Jianbo. The CMU Motion of Body (MoBo) Database. Pittsburgh, Pennsylvania: Carnegie Mellon University, 2001

[21] http://idlastro.gsfc.nasa.gov/idl html help/RADON.html

[22] L. Chen, Yunhong Wang, Yiding Wang, and De Zhang; "Gender Recognition from Gait using Radon Transform and Relevant Component Analysis", International Conference on Intelligent Computing, pp. 92-101, 2009.

[23] E. Murat, "Human Identification Using Gait", Turk J Elec Engin, VOL.14, NO.2 2006, c T"UB_ITAK

[24] M.Siva Sangari and B.Mathivanan; "Performance Evaluation of LDA \& RADON in GAIT Recognition”, International Journal of Computer Applications (0975 - 8887), Volume 13- No.8, pp: 1-5, January 2011.

A. Hayder, He received the B.Sc. degree in electrical engineering from Multimedia University, Selangor, Malaysia, 2008. He has been working as a research assistant at Universiti Malaysia Sabah (UMS), Malaysia. His present research interests include the biometric and image processing.

(Email: mdhayderali@yahoo.com)

J. Dargham, He received his B.Sc. in Control Systems Engineering from Iraq and his M.Sc. in Control System Engineering (UMIST) from Malaysia. He received PhD from University Malaysia Sabah (UMS). He is holding senior lecture position at University Malaysia Sabah (UMS) and program head of the computer Engineering. His research interests include Source Coding, Pattern Recognition, Medical Imaging, Biometrics, Data Compression, and Artificial Intelligence. (Email: jamalad@ums.edu.my ).

A. Chekima, He received his BEngg in Electronics from Ecole Nationale Polytechnique of Algiers in 1976 and his MSc and PhD both in Electrical Engineering from Rensselaer Polytechnic Institute Troy, New York, in 1979 and 1984 respectively. He joined the Electronics Department at the Ecole Nationale Polytechnique in 1984, where he was Chairman of the Scientific Committee of the Department as well as in charge of the Postgraduate Program while teaching at both graduate and undergraduate levels. He was member of several scientific committees at the national level. He has been working as an Associate Professor at the School of Engineering and Information Technology at Universiti Malaysia Sabah since October 1996. His research interests include Source Coding, Antennas, Signal Processing, Pattern Recognition, Medical Imaging, Biometrics, Data Compression, Artificial Intelligence and Data Mining. He has published more than 120 papers in refereed journals, conferences, book chapters and research reports. (E-mail: chekima@ums.edu.my ).

G.M. Moung, He received the B.Sc. degree in computer engineering from University Malaysia Sabah, Malaysia, 2007. He has been working as a research assistant at Universiti Malaysia Sabah, Malaysia. His present research interests include the biometric and image processing. (Email: menirva.com@gmail.com ) 